\title{
The Use of Test Anxiety Assessment and Anxiety Reduction Training to Predict and Improve Performance of Collegiate Pilot Trainees
}

\author{
Teresa Ann Sloan, Michael Lundin, Dale Wilson, and Randy Robinnette \\ Central Washington University
}

\begin{abstract}
Written, oral, and practical flight tests, along with challenging flying tasks, place pilot trainees in stressful situations. The initial goals of this research were to determine assessment tools for identifying pilot trainees who might perform poorly in stressful flight testing environments, and measure the efficacy of a test anxiety (TA) workshop on anxiety levels and Federal Aviation Administration (FAA) knowledge assessments of pilot trainees. The researchers determined that: Scholastic Achievement Test (SAT) scores marginally predict facilitating anxiety levels, and FAA knowledge tests, taken in authentic testing environments, correlate significantly with debilitating anxiety, facilitating anxiety, and FAA exam scores. The researchers recommend continuing longitudinal assessment of freshman flight students that potentially links anxiety and performance on low-risk assessments with these measures on higher-risk practical flight tests. Further study is needed to determine if a more intense anxiety treatment can lower debilitating anxiety, raise facilitating anxiety, or improve performance on FAA exams.
\end{abstract}

\section{INTRODUCTION}

To ensure satisfactory pilot performance and safe flight operations, flight training involves continuous evaluation of aeronautical knowledge and piloting skills. At the researchers' institution, for example, the Department of Aviation provides a stringent curriculum which includes approved ground and flight training conducted under Title 14 Code of Federal Regulations Part 141. The Department's Federal Aviation Administration (FAA) approved curriculum requirements dictate a minimum passing score of $80 \%$ on ground school stage and end-of-course examinations. Failure to meet these requirements or failure to satisfactorily complete flight training lessons and check rides within the approved time-frame constitute grounds for termination from the flight training program.

Consequences for poor performance on flight evaluations can also be severe. Pilot trainees who perform poorly incur the expense of additional training and check rides and risk termination from the flight training program. Clearly, failed check rides threaten aviation careers, especially in light of new legislation. The Airline Safety and Pilot Training Improvement Act of 2009 (H.R. 3371, 111th Cong., 2009) amended the Pilot Records Improvement Act of 1996 (1997) to require the FAA to maintain records of all failed attempts by a pilot regarding practical flight tests for obtaining a certificate or type rating (Section 6 (b) (2) (i) (2) (A) [ii]). H.R. 3371 states that air carriers must access and evaluate information from pilot records regarding practical flight test failures before allowing pilots to begin service with the carrier (Section 6 (b) (2) (i) [1]), and that they may use such records to assess the qualifications of the individual in deciding whether or not to hire the individual as a pilot (Section 6 (b) (2) (i) (9) [A]). In preparation for a career as an air carrier pilot, Section 11 (c) (2) of H.R. 3371 will require "sufficient flight hours... in difficult operational conditions that may be encountered by an air carrier to enable a pilot to operate safely in such conditions". Ultimately, then, the pressure to perform well during flight training presents pilot trainees with the challenge of learning efficiently while overcoming barriers to that learning.

One such barrier faced by pilot trainees is test anxiety (TA). In a previous, more comprehensive literature review, Sloan and Wilson (2009) cited foundational studies in test anxiety. In part, they found 
that TA can be defined as "an unpleasant feeling or emotional state that has both physiological and behavioral concomitants and that is experienced in formal testing or other evaluative situations" (Dusek, 1980, p. 88). Sarason, Mandler, and Craighill (1952) hypothesized:

When a stimulus situation contains elements which specifically arouse test or achievement anxiety, this increase in anxiety drive will lead to poorer performance in individuals who have test-irrelevant [incompatible or interfering] anxiety responses in their response repertory. For individuals without such response tendencies, these stimulus elements will raise their general drive level and result in improved performance (p. 561).

Formalizing the dual nature of TA suggested above, Alpert and Haber (1960) divided TA into two types: debilitating TA (DTA) which reduces performance, and facilitating TA (FTA) which improves performance. They devised the Achievement Anxiety Test (AAT) to measure an individual's predilection for either or both FTA and DTA. They concluded, "...that the incorporation of items designed to measure facilitating anxiety into a scale which already effectively measures debilitating anxiety can significantly increase the prediction of academic performance scores" (p. 215). The AAT has withstood the test of time. Wright State University and the University of Adelaide provide the AAT on their counseling websites. More recent research also incorporates the use of the AAT (Brewer, 2002; Haynes, 2003; Erickson, Horan, \& Hackett, 1991; Fischer \& Corcoran, 2007; Moyer, 2008; Robinson, 2008; Roney \& Woods, 2003).

In their 2009 literature review, Sloan and Wilson concluded that "students who suffer from debilitating TA often match the performance of their peers in non-testing situations" (p. 12). They also stated that DTA can be self-perpetuating: when students do poorly on one test, they can develop a reduced feeling of self-efficacy which can negatively impact performance on future tests.

Observations by faculty from the host institution, in concert with testimonies of test-anxious pilot trainees, affirm the negative impact of debilitating test anxiety on assessments. Additionally, the faculty noted that debilitating test anxiety manifests itself in physiological and emotional symptoms, such as excessive nervousness or expressions of frustration and inadequacy.

Retaining qualified students in flight programs is desirable. In an introduction to Sloan and Wilson's 2009 literature review, Kohlruss (2009) suggests:

If we can assist our students in succeeding with their FAA check rides we can help them stay in our programs. This of course will motivate them to stay in our industry and not stray off to other disciplines other than aviation. Our industry needs the best and the brightest. We cannot afford to lose them due to stress interfering with their successful performance on a check ride (p. 7).

Consequently, strategies that help pilot trainees cope with anxiety should also help them perform better during program assessments. Better test performance and anxiety coping skills should lead to increased retention in aviation programs and build stronger candidates who continue to withstand the rigors of HR3371 well into their careers.

Regarding training designed to reduce DTA, Sloan and Wilson (2009) also concluded "the progression of TA research shows that no single treatment method was superior, and a combination approach of cognitive, behavioral, and study skills treatments had the greatest effectiveness" (p. 12). They proposed that, "a single day workshop appeared to be as effective as sessions conducted over a longer period and is more apt to attract both participants and facilitators" (p. 12). 
The purpose of this present study is: (a) to determine if the AAT, when adjusted with total Scholastic Assessment Test (SAT) scores (a long standing predictor of success in college [The College Board, n.d.]), is an accurate predictor of performance; and (b) to see if a TA workshop reduces DTA, increases FTA, or increases performance on aviation knowledge assessments.

\section{METHOD}

\section{Participants}

Out of 103 pilot trainees enrolled in a flight technology major at the host institution and actively pursuing flight training (91 men and 12 women, mean age $=20.95$ years), 33 volunteered to participate in the study (27 men and 6 women). Volunteers' academic and flight experience ranged from freshmen to senior class standing and from student pilot to commercial pilot. Volunteers were assessed for DTA using the AAT. Positive DTA scores were identified as ranging from 21 through $36(-1 /+1.5$ sd from the mean of 26.6), and only those who tested positive $(n=26 ; 22$ men and 4 women $)$ were selected as participants. Six volunteers were identified as not being test anxious due to their scores, and one volunteer who scored high on the TA scale (38) was eliminated due to suspicion of faulty testing. Participants were assigned to one of four levels (private, instrument, commercial, or certified flight instructor [CFI] according to their last ground school enrollment). Each level was then randomly assigned to the treatment group (total $\mathrm{n}=$ 13; private $\mathrm{n}=3$, commercial $\mathrm{n}=5$; instrument $\mathrm{n}=3$; CFI $\mathrm{n}=2$ ) and control group (total $\mathrm{n}=13$; private $\mathrm{n}=3$; commercial $\mathrm{n}=4$; instrument $\mathrm{n}=4$; CFI $\mathrm{n}=2$ ).

\section{Procedure}

Following the selection of participants, both the control and treatment groups were administered the AAT and an FAA knowledge pre-test. One week later the treatment group participated in a three-hour TA workshop conducted by an experienced counseling psychologist employed by the University's Health and Counseling Center and trained in TA reduction techniques. The following week, participants from both groups retook the AAT and completed a second appropriate FAA knowledge post-test.

The key elements of the performance anxiety workshop included psychoeducation and experiential exercises. The psychoeducational element included discussing the nature and function of anxiety, how anxious thoughts are related to anxious feelings, how anxiety undermines attention and performance, and the futility of struggling to make anxiety go away. The experiential exercises included a self-assessment of anxious experiences, metaphors emphasizing the idea that the more an individual struggles with anxiety the more present it is, and a mindfulness exercise. The workshop concluded with the recommendation that the participants practice self-observation and mindful acceptance in situations that provoke anxious experiences other than test situations. The last recommendation was made in order to enhance self-regulation.

\section{Instruments}

In addition to the AAT, the researchers employed other measures of college readiness and aviation knowledge in their analysis. These measures included FAA aeronautical knowledge test (AKT) scores from the most recent FAA exam taken by the participants, SAT scores, and the host institution's Admissions Index (AI) - a probability of success indicator that takes into account high school grade point average (GPA) and standardized test scores. Notably, AKT scores came from four different tests, depending on participants' level: private pilot, instrument pilot, commercial pilot, or CFI. SAT scores were not available for 5 transfer students. 
Additionally, four levels of AKTs, consisting of 15 questions each, were created from the FAA knowledge test-question data base. More specifically, the researchers constructed pre- and post-treatment private, instrument, commercial, and CFI tests for two reasons: to instigate authentic test-taking scenarios, and to measure change in performance levels after treatment. The post-treatment tests consisted of different questions on the same general knowledge areas used on the pre-treatment tests.

\section{RESULTS}

The researchers' goals were: (1) to determine assessment tools for identifying pilot trainees who might perform poorly in stressful flight testing environments, and (2) to measure the efficacy of a test anxiety workshop treatment on pilot trainees' anxiety levels and their performance on FAA knowledge assessments. The level of significance for all statistical tests herein was $\alpha=.05$.

\section{Identifying Pilot Trainees Prone to Poor Flight Testing Performance Due to Anxiety}

The researchers took two courses of action toward identifying DTA prone pilot trainees: First, they identified college readiness test data that might correlate with anxiety levels, and second, they issued preand post-debilitating and facilitating anxiety tests, respectively, before and after treatment. To tease out relationships among college readiness measures, anxiety measures, and knowledge measures, the researchers ran a $10 \times 10$ bivariate correlation matrix (Table 1) with variables: facilitating anxiety pretreatment test (FTA1), facilitating anxiety post-treatment test (FTA2), debilitating anxiety pre-treatment test (DTA1), debilitating anxiety post-treatment test (DTA2), FAA knowledge pre-treatment test (FAA1), FAA knowledge post-treatment test (FAA2), Admissions Index (AI), Scholastic Achievement Test (SAT), FAA class final (CF), and FAA aeronautical knowledge test (AKT). Results indicated that SAT correlated marginally with FTA1 and FTA2 measures, and significantly with the FAA2 variable. AI, the host institution's measure of college readiness, correlated (highly) with only one measure of importance: the FAA1 variable. Furthermore, neither DTA1 nor DTA2 variables correlated significantly with either SAT or AI. The researchers concluded that SAT scores may help predict FTA due to positive but marginally significant correlation.

The AKT provided an authentic benchmark against which to profile by correlation other knowledge variables (see Table 1). The AKT correlated significantly (and with correct valences) with debilitating anxiety measures, FAA knowledge pre- and post-treatment test scores, CF scores, and AI. Evidently, FAA2 was the more realistic testing situation from which to measure anxiety, compared with the FAA1. The FAA2 correlation profile in Table 1 affirms its authentic testing character, comparing favorably with that of the benchmark AKT. In contrast, the FAA1 correlated significantly but highly $(\mathrm{p}<.001, \mathrm{r}=.798)$ only with the AI variable, the preferred predictor of first-year performance at the host institution. Because AI is a linear combination of SAT and high school GPA measures, the researchers inferred that the GPA component "dropped out" as a predictor of performance for FAA2. Such an assumption suggests that FAA2 experience was beyond the high school core, affirming the FAA2 test-taking scenario to be more anxiety producing and therefore, more authentic. In any case, the above statistical results led researchers to conclude that the FAA2 testing scenario was authentic, while the FAA1 scenario lacked authenticity. 
Table 1. Bivariate Correlation Profiles for Anxiety, Knowledge, and Predictor Variables

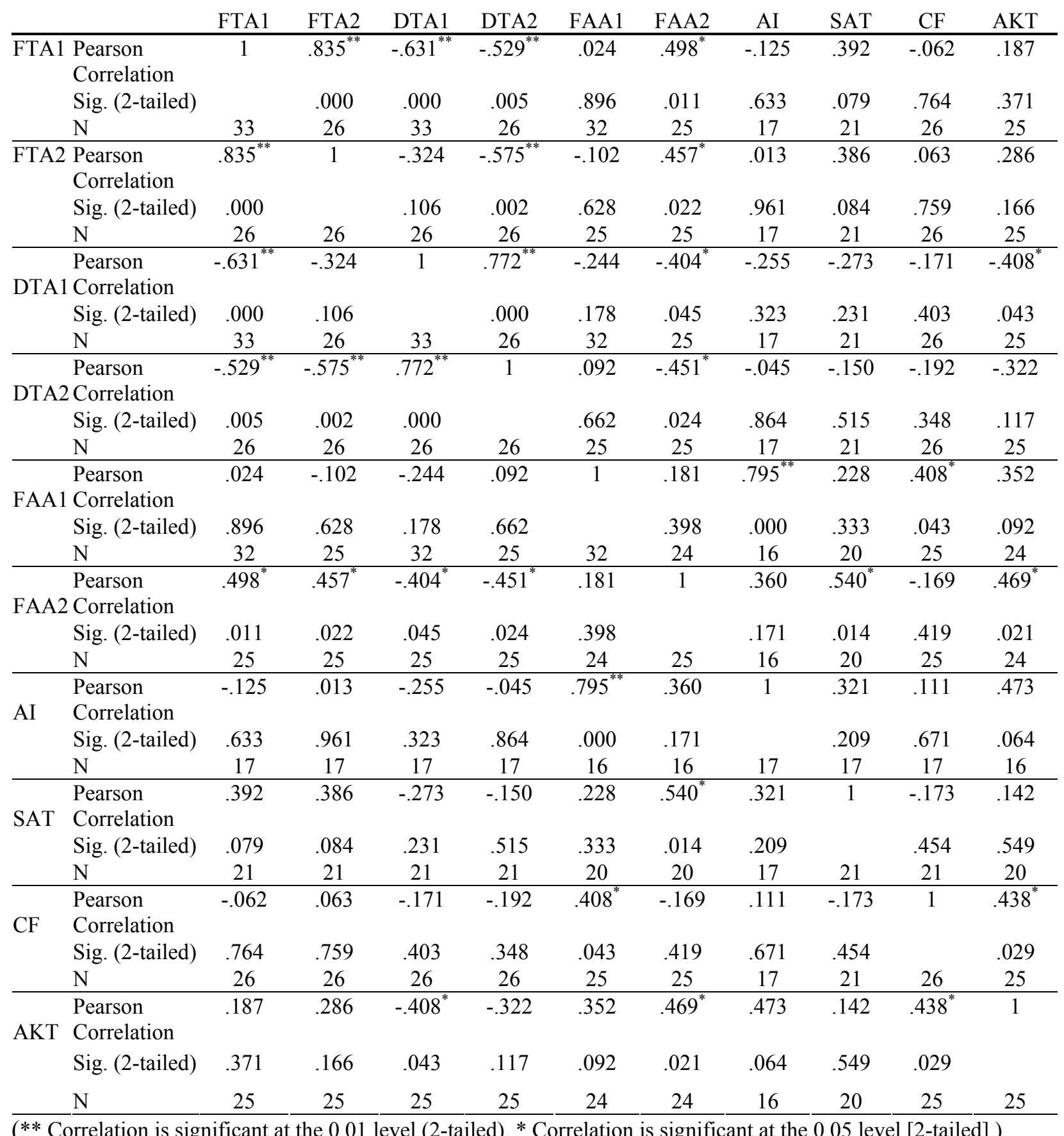

(** Correlation is significant at the 0.01 level (2-tailed). * Correlation is significant at the 0.05 level [2-tailed].)

\section{Measuring the Efficacy of the Anxiety Workshop on Pilot Trainees' Knowledge Assessments}

The researchers decided against attempting to capture the effects of the anxiety workshop on knowledge assessments, based on too little data. The number who participated in the TA workshop was low $(\mathrm{n}=13)$. A larger sample may have yielded different results. Moreover, FAA1 and FAA2 scores 
spanned four different levels, making any conclusions about significant improvements suspect. Initially, FAA1 and FAA2 were thought to capture enough information to reflect the efficacy of the anxiety workshop. However, statistical scans of the data showed little evidence for including pre-treatment or post-treatment knowledge test variables into a main model. Instead the main linear model, discussed below, included only anxiety measures as dependent variables.

\section{Measuring the Efficacy of the Anxiety Workshop on Pilot Trainees' Anxiety Levels}

The researchers wished to determine if participants' facilitating or debilitating anxiety levels significantly changed as a result of treatment when their responses to anxiety assessments were factored by pre- or post- levels and treatment or control levels (see Table 2). Dependent variables were anxiety measures FTA and DTA and independent variables were FTA Factor, DTA Factor, and Group Factor. FTA factor had pre- and post- levels, respectively: FTA1 and FTA2. DTA had pre- and post- levels, respectively: DTA1 and DTA2. The Independent variable Group had two levels: Treatment and Control. There was a single covariate SAT, chosen because it correlated with the most variables of interest: AI, FAA2, and measures of facilitating anxiety (FTA1 and FTA2).

Table 2. Factorial Model

\begin{tabular}{ccc}
\hline Dependent Variables & Independent Variables & Covariate \\
\hline FTA & Within-Subjects Factor Levels & SAT \\
DTA & FTA1 (pre-test) & \\
& FTA2 (post-test & \\
DTA1 (pre-test) & \\
DTA2 (post-test) & \\
& Between-Subjects Factor Levels & \\
& Group & \\
Treatment & \\
& Control & \\
\hline
\end{tabular}

\section{Results of LMRMA Omnibus Test}

To test for the significance of contributions of variables, the researchers employed a Linear Multivariate Repeated-Measure Analysis (LMRMA). Table 3 shows descriptive statistics for the sample, disaggregated by eight factor levels. The multivariate Wilk's Lambda test indicated significant contributions to the linear model by FTA and interaction variables FTA*SAT and FTA*Group only (see Table 4). Notably, the dependent variable DTA made no significant contribution to the LMRMA model (Wilk's $\Lambda=.993, \mathrm{~F}(1,18)=.135$, and $\mathrm{p}=.717$ ), nor did interactions of DTA*Group or DTA*SAT. Importantly, the significant interaction FTA*SAT suggested inequitable adjustments to FTA values when comparing the contributions of FTA1 and FTA2. Such adjustments by the covariate at unequal rates to levels FTA1 and FTA2 compromise any conclusions about the change in facilitating anxiety before and after treatment. Finally, the significant interaction of FTA*Group pointed to the need for more analysis. 
Table 3. Descriptive Statistics for the Factorial Model

\begin{tabular}{lccc}
\hline Factor/Level & Mean & Std. Deviation & $\mathrm{N}$ \\
\hline FTA1 & & & \\
$\quad$ Control & 24.00 & 4.295 & 10 \\
$\quad$ Treatment & 27.82 & 5.510 & 11 \\
FTA2 & & & \\
$\quad$ Control & 24.70 & 6.038 & 10 \\
$\quad$ Treatment & 27.27 & 4.519 & 11 \\
DTA1 & & & \\
Control & 28.80 & 4.211 & 10 \\
$\quad$ Treatment & 27.82 & 4.468 & 11 \\
DTA2 & & & \\
Control & 29.00 & 5.375 & 10 \\
Treatment & 28.18 & 3.737 & 11
\end{tabular}

Keeping with best practice, the covariate SAT was removed from the LMRMA model, because the pathological interaction FTA*SAT suggested inequitable adjustments to means. Hence, conclusions about significant differences, suggested in Table 4 lacked validity. Without covariate adjustment, however, all LMRMA significance collapsed upon rerunning the model. The collapse of significance upon removal of the SAT covariate suggested an association between SAT and FTA variables. This evidence, coupled with the positive correlations between SAT and Facilitating Anxiety variables adds credence to a conjecture: SAT scores predict facilitating anxiety.

Table 4. Significant Statistics from the LMRMA

\begin{tabular}{lccc}
\hline & Wilks $\Lambda$ & $\mathrm{F}(1,18)$ & $\mathrm{p}$ \\
\hline FTA & .700 & 7.725 & .012 \\
FTA*SAT & .732 & 6.581 & .019 \\
FTA*Group & .792 & 4.740 & .043 \\
DTA & .993 & .135 & .717 \\
\hline
\end{tabular}

\section{DISCUSSION}

The shorter periods between pre-tests, treatment, and post-tests may not have provided the time needed to practice the strategies that had been taught in the workshop nor sufficient time for forgetting. The length of the workshop was reduced from one-day to three hours (the research originally was marketed with a one day workshop, but even fewer volunteers $[\mathrm{n}=6]$ agreed to enter the research). Regardless, the researchers believe the duration and intensity of an effective workshop warrants further study. Finally, one researcher suggested that the lack of efficacy of the treatment might be related to three personal factors: (a) appropriate study skills, (b) cognitive ability, and (c) motivation to sacrifice in order 
to successfully reach goals. Students with high motivation and moderate ability may be successful, whereas students with high motivation but low ability might not be successful despite their efforts. The effect of personal factors on performance and anxiety levels warrants further study.

SAT scores were positively associated with FTA test scores, but marginally so. Although SAT scores significantly correlated with FTA, as a covariate in the main linear model of this study, they adjusted FTA1 and FTA2 scores at different rates. Consequently, SAT scores were removed from the main model. When SAT scores were removed as a covariate, the main model of this study no longer showed any significant differences between variables of interest. However, clear evidence exists that SAT scores are related marginally to FTA1 and FTA2 and significantly to FAA2. More research is needed to sort out the complex interaction among college preparation, anxiety and performance measures. Evidence herein begs for such a study, hinting that SAT scores rise and fall with students' facilitating anxiety levels.

Authentic testing situations are important when attempting to discern anxiety levels. Authenticity is in part detected by correlation among the variables of interest. Researchers found that the post-treatment knowledge test (FAA2) significantly correlated with both the pre- and post-facilitating test anxiety (FTA1 and FTA2) scores, both pre- and post-debilitating test anxiety (DTA1 and DTA2) scores, SAT scores, and, most importantly, the FAA aeronautical knowledge test (AKT). By association, then, the researchers deemed the FAA2 testing scenario as authentic. However, the FAA1 test only associated with the AI, lacking associations with other variables that would affirm its authenticity in a testing scenario. The important lesson here is that authentic testing scenarios are necessary to glean information about treatment effects during further studies.

These results suggest that the AAT, combined with the SAT, can be used by collegiate aviation educators to identify flight training students predisposed to high DTA and low FTA. Early identification of vulnerable students through the use of these assessments and effective mitigation strategies designed to help them overcome the deleterious effects of DTA could help pilot trainees achieve their educational and flight training goals. Importantly, AAT screening should be done in the context of authentic testing scenarios to guarantee that anxiety reactions are appropriate to stressors.

\section{RECOMMENDATIONS}

The researchers recommend and plan further research to confirm their conclusion that the SAT and AAT are accurate predictors of performance. In order to incorporate more authentic anxiety-producing test methods, they recommend administering the AAT to all incoming freshmen private pilot students during authentic testing scenarios. AATs should be administered before each of three ground training stage checks. The results of these AATs will be compared with pilot trainees' ground and flight stage check scores to determine correlations between test anxiety and performance, both in the classroom and in the cockpit. Testing should be repeated over a two to three year period.

In conclusion, anxiety and flight test performance are inextricably linked. However, that link is complex. Further study should result in more accurate detection and intervention treatments for testanxious pilot trainees. The researchers hope to establish a program of TA detection and intervention, documenting the positive effects of a treatment on classroom, ground, and flight performance. 


\section{REFERENCES}

Airline Safety and Pilot Training Improvement Act of 2009, H.R. 3371, 111th Cong. (2009). Retrieved from http://www.govtrack.us/congress/billtext.xpd?bill=h111-3371

Alpert, R., \& Haber, R. N. (1960). Anxiety in academic achievement situations. Journal of Abnormal and Social Psychology, 61(2), 107-215.

Brewer, T. (2002). Test taking anxiety among nursing and general college students. Journal of Psychosocial Nursing, 40(11), 23-29.

Dusek, J. B. (1980). The development of test anxiety in children. In I. G. Sarason (Ed.), Test anxiety: Theory, research, and applications (pp. 87-110). Hillsdale, NJ: Erlbaum.

Erickson, C. D., Horan, J. J., \& Hackett, G. (August 1991). On thinking and feeling bad: Do client problems derive from a common irrationality or specific irrational beliefs? Paper presented at the annual meeting of the American Psychological Association, San Francisco, CA.

Fischer, J. \& Corcoran, K. (2007). Measures for clinical practice and research: a sourcebook. (4th ed). Oxford, England: Oxford University Press.

Haynes, S. E. (2003). The effect of background music on the mathematics test anxiety of college algebra students. (Doctoral dissertation). Retrieved from http://wvuscholar.wvu.edu:8881//exlibris/dtl/d3_1/apache_media/6747.pdf

Kohlruss, W. A. (2009). The editor's forum. The Journal of Aviation/Aerospace Education and Research, 19(1), 7.

Moyer, K. H. (2008). Debilitating and facilitating anxiety effects on identification. Journal of Undergraduate Psychological Research. 3, 6-20.

Pilot Records Improvement Act of 1996, 49 U.S.C. $§ 44703$ (h). (1997).

Robinson, D. H. (2008). Evaluation (test) anxiety. In E. M. Anderman \& L. Anderman (Eds.), Psychology of classroom learning: an encyclopedia (pp. 387-390). Farmington Hills, MI: Thomson Gale.

Roney, S. D. \& Woods, D. R. (2003). Ideas to minimize exam anxiety. Journal of Engineering Education, 92(3), 249-256.

Sarason, S. B., Mandler, G., \& Craighill, P. G. (1952). The effect of differential instructions on anxiety and learning. Journal of Abnormal Psychology, 47, 561-565.

Sloan, T. A. \& Wilson, D. (2009). Assessment and intervention strategies for test anxiety in aviation students. The Journal of Aviation/Aerospace Education and Research, 19(1), 9-14.

The College Board. (n.d). Retrieved from http://professionals.collegeboard.com/testing/sat-reasoning

University of Adelaide (n.d.). Retrieved from http://www.adelaide.edu.au/counselling)centre/Test.html

Wright State University Counseling Center (n.d.) Retrieved from http://www.wrightcounseling.com/checklists/TestAnxietyAssessment.html 